\title{
The influence of Stable Stratification on the Transition to Turbulence in a Temporal Mixing Layer
}

Fundação Universidade Federal do Rio Grande 96201-900 Rio Grande, RS, Brazil

\section{Edith Beatriz C. Schettini}

bcamano@iph.ufrgs.br

Instituto de Pesquisas Hidráulicas

Universidade Federal do Rio Grande do Sul

Cx. Postal 15029

91501-970 Porto Alegre, RS, Brazil

\section{Jorge Hugo Silvestrini}

Senior Member, ABCM

jorgehs@pucrs.br

Departamento de Eng. Mecânica e Mecatrônica

Pontifícia Univ. Católica do Rio Grande do Sul 906190-900 Porto Alegre, RS, Brazil
The transition to turbulence in a stably stratified flow is a problem of considerable interest in fluid dynamics with applications in both geophysical sciences and engineering. This transition is controlled by competition between the vertical shear of the base flow and the buoyancy forces due to the density stratification. The present work investigates numerically the effect of stable stratification on the development of a Kelvin-Helmholtz. $(K H)$ instability and the formation of streamwise vortices, which are developed after the saturation of the primary billows of KH. The Direct Numerical Simulation (DNS) technique was used to solve the complete Navier-Stokes equations, in the Boussinesq approximation. Numerical tests were done with different Richardson numbers and forced initial conditions for velocity fluctuations. The results showed that high stratification inhibits the pairing process, reduces the buoyancy flux, weakens the vertical motions, decreases the thickness of the mixing layer and affects the formation of streamwise vortices. The three-dimensional results demonstrated that the streamwise vortices are clearly formed in non-stratified cases. In the stratified cases, on the other hand, the streamwise vortices are weakened, due to the streamwise density gradient, which decrease the levels of vorticity in the billows of $K H$, while increase in the braid zone.

Keywords: Stable stratification, direct numerical simulation, mixing layer, buoyancy force, transition to turbulence

\section{Introduction}

From a fundamental point of view, the transition to turbulence in a stably stratified flow is interesting because it plays a key role in better understanding the mixing and dynamical processes in environmental or industrial problems.

The dynamics of the stably stratified mixing layer is controlled by a competition between the vertical shear of the base flow and the buoyancy forces due to the density stratification. The buoyancy effects act reducing the growth rate of perturbation and delaying the transition to turbulence, while the shear supplies kinetic energy to the flow. The evolution of such flows is commonly studied in terms of a model problem: the mixing layer. The stably stratified mixing layer develops at the interface of two parallel streams of fluid moving horizontally at different velocities and having different densities, the upper stream being lighter than the lower one.

Miles (1961) and Howard (1961), based on a linear stability analysis, showed that for the Kelvin-Helmholtz $(\mathrm{KH})$ instability to occur in stratified mixing layer from an infinitely small disturbance, the Richardson number must be less than 0.25 somewhere within the flow. This first instability that occurs in the mixing layer is due to the inflectional nature of the velocity profile (Michalke, 1964): the vortex sheet initially created is linearly unstable and rolls up to form the primary billows of $\mathrm{KH}$.

The second stage of the transition in the mixing layer occurs due to the formation of streamwise vortices (known as "ribs" vortices), which develop after the primary billows of KH. The transition to turbulence in the non-stratified mixing layer has been widely studied in the last two decades (Lasheras and Choi, 1988; Rogers and Moser, 1992; Comte et al., 1992; Moser and Rogers, 1993; Comte et al., 1998).

Pierrehumbert and Widnall (1982) found that a periodic array of Stuart vortices, with a similar configuration of $\mathrm{KH}$ vortices, is

Presented at ETT $2004-4^{\text {th }}$ Spring School on Transition and Turbulence September $27^{\text {th }}$ - October $1^{\text {st }}, 2004$, Porto Alegre, RS, Brazil.

Paper accepted: May, 2005. Technical Editor: Aristeu da Silveira Neto. unstable to different three-dimensional (3D) disturbances. They demonstrated that this unstable mode is characterized by spanwise oscillation in phase with primary billows of KH. This instability, called translative, is well known in the literature as being the responsible for the beginning of the three-dimensionality in the nonstratified mixing layer and, consequently, for the formation of streamwise vortices. The most unstable spanwise wavelength $\left(\lambda_{y}\right)$ for a translative instability was found to be $2 / 3$ of the separation between $\mathrm{KH}$ vortices (streamwise wavelength, $\lambda_{\mathrm{x}}$ ).

Metcalfe et al. (1987) studied through Direct Numerical Simulation (DNS) the evolution of a non-stratified mixing layer $(\mathrm{Ri}=0.0)$ and investigated a secondary instability induced by different initial velocity conditions. They showed that the streamwise vortices are characteristic structures of 3D mixing layers and its generation depends strongly on the initial condition.

In stratified mixing layers, the 3D process is more complex than in non-stratified mixing layers. This fact is due to the greater number of secondary instabilities that propagate in the flow. The instabilities that develop in a 3D stably stratified mixing layer may be divided in two groups: one that grows within the vortex core and the other that develops in the region between the cores (the braids). Within the cores two types of instabilities are found. The one discovered by Pierrehumbert and Widnall (1982) that does not depend upon the buoyancy effects and the gravitational convective instability that is driven by buoyancy effects. The other that develops in the region between the cores was predicted by Klaassen and Peltier (1991) and verified by Schowalter et al. (1994) in laboratory experiments. The instability that grows in the braids is called secondary shear instability (Caulfield and Peltier, 1994).

The gravitational convective instability and the secondary shear instability that are restricted to a stratified mixing layer are caused by the streamwise density gradient imposed by the buoyancy force. The gravitational convective instability makes unstable the sub layers of density generated during the roll-up of the $\mathrm{KH}$ billows. The secondary shear instability induced by the streamwise density gradient develops in the braid region between two KelvinHelmholtz vortices. The presence of secondary shear instability in a stratified mixing layer is due to baroclinic vorticity generation given by a source term $\left(-g / \rho_{0} \partial \rho / \partial x\right)$, which concentrates the vorticity in 
the baroclinic layer (Staquet, 1995). Thus, the density gradient source term contributes as an extra mechanism for the generation or destruction of local vorticity by means of the baroclinic torque (Caulfield and Peltier, 2000; Cortesi et al., 1998).

The Richardson number effects on the secondary shear instability has been studied in some detail by Klaassen and Peltier (1991) by performing stability analyses in the linear range. The strongest growth-rate for this kind of instability was found to occur between $R i=0.08$ and $R i=0.12$, and to approach zero growth rate at $R i=0.22$, which is close to the critical inviscid value, $R i=0.25$, for the primary KH instability (Miles, 1961). They found that the most unstable spanwise wavelength is smaller than the streamwise one, for $R i=0.0$ to 0.04 , whereas for higher stratification the wavelength does not vary significantly.

Schowalter et al. (1994) investigated experimentally the threedimensionalization of a free shear layer for different Richardson numbers, with forced condition. They noticed that the streamwise vortices were weakened or reinforced by stratification, depending on their location. On the top the KH billows, when unstably stratified regions were formed (heavy fluid above, $(\partial \rho / \partial z<0)$ ), strong streamwise vorticity was measured, while in the braids the ribs were weak.

The present work investigates the nature of transition to turbulence in a stably stratified temporal mixing layer through the direct numerical simulation (DNS). The main objective of this work is to analyze the influence of a stable stratification on the development of the $\mathrm{KH}$ instability and in the formation of streamwise vortices. In this study it is shown that the stratification affects the formation of streamwise vortices, even when forced conditions are used. The DNS technique is used to solve the complete Navier-Stokes equations, in the Boussinesq approximation. Direct numerical simulations are excellent instruments for the investigation of the dynamics of a stably stratified mixing layer, since they solve entirely all the spatial and temporal scales of the flow.

In the following sections first the governing equations and the numerical method are presented. Then, code validation test cases are presented comparing the DNS results with results from the linear stability theory. The following section describes the results from the two-dimensional and three-dimensional simulations, for different Richardson numbers $(R i=0 ; 0.1 ; 0.2)$, showing the evolution of the mixing layer, with the formation of baroclinic layer and of the streamwise vortices, respectively. Finally the conclusions and final comments are presented.

\section{Nomenclature}

$R i=$ Richardson number .

$R e=$ Reynolds number.

$R=$ Ratio of the initial vorticity thickness to the density thickness.

$U=$ Reference velocity.

$P=$ Pressure.

$t=$ Time.

$\boldsymbol{u}=$ Velocity field.

$x, y, z=$ Streamwise, spanwise and vertical coordinate directions.

$u, v, w=$ Streamwise, spanwise and vertical velocity components.

Pr $=$ Prandtl number.

$L_{x}, L_{y}, L_{z}=$ Streamwise, spanwise and vertical domain length. $g=$ Acceleration due to gravity.

$n_{x}, n_{y}, n_{z}=$ Number of points in the streamwise, spanwise and vertical directions.

\section{Greek Symbols}

$\rho(x, y, z, t)=$ Density or active scalar.

$\rho_{0}=$ Reference density.

$\Delta \rho=$ Density difference across the shear layer.

$\delta_{i}=$ Initial vorticity thickness.

$\delta_{i} / U=$ Advective time scale.

$\delta_{d}=$ Density thickness .

$\alpha_{a}=$ The most amplified wavenumber.

$\lambda_{a}=$ The most unstable wavelength .

$\lambda_{I}=$ Fundamental wavelength.

$\lambda_{x}, \lambda_{y}=$ Streamwise wavelength, spanwise wavelength.

$v=$ Kinematic viscosity.

\section{Formulation and Numerical Method}

\section{Governing Equations}

The equations that govern the fluid motion are the NavierStokes equations with the Boussinesq approximation, in a Cartesian frame of reference $\Re=(0 ; x, y, z)$. The momentum equation for the velocity field $\boldsymbol{u}$, with components $(u, v, w)$, is given by:

$\frac{\partial \boldsymbol{u}}{\partial t}=-\nabla P-\boldsymbol{\omega} \times \boldsymbol{u}-R i \rho \boldsymbol{i}_{z}+\frac{1}{R e} \nabla^{2} \boldsymbol{u}$

where $P=p+\left(\rho \boldsymbol{u}^{2} / 2\right)$ is the modified pressure field.

The continuity equation is:

$\nabla \cdot \boldsymbol{u}=0$,

and the transport equation is:

$\frac{\partial \rho}{\partial t}+(\boldsymbol{u} \cdot \nabla) \rho=\frac{1}{\operatorname{RePr}} \nabla^{2} \rho$.

The variables used in the above equations are non-dimensional. There are two non-dimensional relevant parameters: the Reynolds and the Richardson numbers. The Reynolds number, based on the half velocity difference across the shear layer $(U)$ and on the initial vorticity thickness $\left(\delta_{i}\right)$, is defined by:

$R e=\frac{U \delta_{i}}{v}$,

where

$\delta_{i}=\frac{2 U}{(d u / d z)_{\max }}$.

The Richardson number is defined by:

$R i=\frac{g R \Delta \rho \delta_{i}}{\rho_{0} U^{2}}$

In these equations, the scales of length, velocity and density are such that $\delta_{i}=1, U=1$ and $\Delta \rho=1 / R$. In this manner, $R e=1 / \nu$ and $R i=g$ $/ \rho_{0}$. 


\section{Initial and Boundary Conditions}

The initial conditions are defined in terms of the velocity and density fields as in Corcos and Sherman (1976) and Patnaik et al. (1976). At the initial time $(t=0)$ the density field is $\rho(x, y, z, t=0)=$ $\rho_{0}+\rho(z)$. In the present case, no density fluctuation is superimposed upon $\rho(z)$ at $t=0$. The initial profiles of velocity and density, at $t=$ 0 are:

$u(z, t=0)=U \operatorname{erf}\left(\frac{\sqrt{\pi} z}{\delta_{i}}\right)$

$\rho(z, t=0)=-\frac{1}{R} \operatorname{erf}\left(\frac{\sqrt{\pi} R z}{\delta_{i}}\right)$

With the objective to promote the development of the $\mathrm{KH}$ instability and to unchain the formation of the KH billows, a field of perturbations was added to the basic velocity profile. This field is composed by two waves corresponding to the most amplified wave number $\left(\alpha_{a}\right)$ and its firth sub-harmonic $\left(\alpha_{a} / 2\right)$, and a sinusoidal wave in spanwise direction. The associated most unstable wavelength given by the linear stability theory is approximately $\lambda_{\mathrm{a}}=7 \delta_{i}$ where the most amplified wave number $\alpha_{\mathrm{a}}=2 \pi / \lambda_{\mathrm{a}}$ being $0.889 \delta_{i}^{-1}$, Michalke, (1964). These perturbations promote, respectively, the development of the $\mathrm{KH}$ instability, the pairing process and the formation of streamwise vortices.

The boundary conditions for the temporal mixing layer are: -periodic: used in the streamwise $(x)$ and spanwise $(y)$ directions; and

-free-slip: used in the vertical direction $(z)$. This condition imposes the following restrictions: $\partial u / \partial z=\partial v / \partial z=0$ and $\mathrm{w}=0$ for $z= \pm L_{z} / 2$.

\section{Numerical Method}

Equations (1) to (3) are solved numerically, in the domain shown in Fig. 1, using a sixth-order compact finite difference scheme (Lele, 1992) to evaluate spatial derivatives.

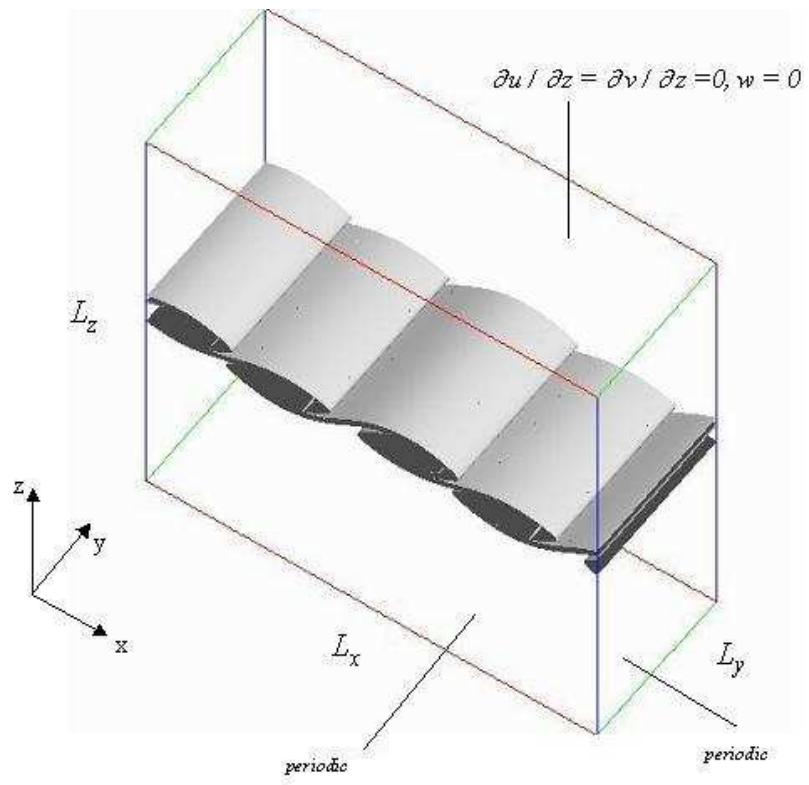

Figure 1. Schematic view of the domain.
The compact schemes are implicit ones that relate the value of the derivative in a point to the value of the derivative in the neighboring points.

For the spatial discretization considering a uniform mesh, where the independent variable for each node $i$ is $\xi_{i}=(i-1) \Delta \xi, 1 \leq i \leq N$ and $\xi=x, y$ or $z$, the function values for the nodes are $f_{i}=f\left(\xi_{i}\right)$ and the first derivative $f^{\prime}{ }_{i}=f^{\prime}\left(\xi_{i}\right)$ is given by:

$$
\begin{gathered}
\alpha f_{i-1}^{\prime}+f_{i}^{\prime}+\alpha f_{i+1}^{\prime}= \\
a \frac{f_{i+1}-f_{i-1}}{2 \Delta \xi}+b \frac{f_{i+2}-f_{i-2}}{4 \Delta \xi} .
\end{gathered}
$$

The second derivative is given by:

$$
\begin{gathered}
\alpha f_{i-1}^{\prime \prime}+f_{i}^{\prime \prime}+\alpha f_{i+1}^{\prime \prime}= \\
a \frac{f_{i+1}-2 f_{i}+f_{i-1}}{\Delta \xi^{2}}+b \frac{f_{i+2}-2 f_{i}+f_{i-2}}{4 \Delta \xi^{2}} .
\end{gathered}
$$

The sixth order is obtained with the set of parameters (Lele, 1992):

$$
\begin{array}{llll}
\alpha=\frac{1}{3}, & a=\frac{14}{9}, & b=\frac{1}{9} & \text { for Eq. (9), } \\
\alpha=\frac{2}{11}, & a=\frac{12}{11}, & b=\frac{3}{11} & \text { for Eq. (10). }
\end{array}
$$

Equations (9) and (10) are valid for the three spatial directions $(x, y, z)$ in all the mesh points.

The time integration is performed with a third-order low-storage Runge-Kutta method (Williamson, 1980). The integration of Eq. (1) at times $t^{n}$ and $t^{(n+1)}$ is performed through three fractional time steps $p=0,1,2$, where $\boldsymbol{u}^{(0)}=\boldsymbol{u}^{(n)}$ and $\boldsymbol{u}^{(3)}=\boldsymbol{u}^{(n+1)}$,

$$
\begin{gathered}
\frac{u^{(p+1)}-u^{(p)}}{\Delta t}= \\
\alpha_{p} F^{(p)}+\beta_{p} F^{(p+1)}-\nabla \Pi^{(p+1)}, \\
\nabla \cdot \boldsymbol{u}^{(p+1)}=0,
\end{gathered}
$$

where

$$
\begin{aligned}
& \boldsymbol{F}=-\boldsymbol{\omega} \times \boldsymbol{u}-\operatorname{Ri} \rho \boldsymbol{i}_{z}+\frac{1}{R e} \nabla^{2} \boldsymbol{u}, \\
& \Pi^{(p+1)}=\frac{1}{\Delta t} \int_{t_{n}}^{t_{(n+1)}} P d t
\end{aligned}
$$

and $\alpha_{p}, \beta_{p}$, are coefficients to each fractional step $p$, given by Williamson (1980):

$$
\begin{array}{ll}
\alpha_{0}=\frac{8}{15}, & \beta_{0}=0 ; \\
\alpha_{1}=\frac{5}{12}, & \beta_{1}=\frac{-17}{60} ; \\
\alpha_{2}=\frac{3}{4}, & \beta_{2}=\frac{-5}{12} .
\end{array}
$$

The Eq. (11) can be split into two steps, 


$$
\begin{aligned}
& \frac{\boldsymbol{u}^{*}-\boldsymbol{u}^{(p)}}{\Delta t}=\alpha_{p} \boldsymbol{F}^{(p)}+\beta_{p} \boldsymbol{F}^{(p-1)}, \\
& \frac{\boldsymbol{u}^{(p+1)}-\boldsymbol{u}^{*}}{\Delta t}=-\nabla \Pi^{(p+1)}
\end{aligned}
$$

In this conventional fractional method, step $p+1$ is obtained by solving the Poisson equation. Thus, the incompressibility condition is ensured as follows,

$$
\nabla \cdot \nabla \Pi^{(p+1)}=\frac{\nabla \cdot \boldsymbol{u}}{\Delta t}
$$

More details about the numerical code can be found in Lardeau et al. (2002) and Silvestrini et al. (2002).

Equation (3) is solved in the same way as Eq. (1) by making,

$$
\frac{\rho^{(p+1)}-\rho^{(p)}}{\Delta \boldsymbol{t}}=\alpha_{p} \boldsymbol{G}^{(p)}+\beta_{p} \boldsymbol{G}^{(p+1)},
$$

where

$$
\boldsymbol{G}=-(\boldsymbol{u} . \nabla) \rho+\frac{1}{\operatorname{RePr}} \nabla^{2} \rho .
$$

\section{Code Verification - Amplification Rate}

In order to validate the numerical code the evolution of a small disturbance was considered in a $2 \mathrm{D}$ domain. The results were compared with the linear stability theory, where the disturbance is described by the Taylor-Goldstein equation (Hazel, 1972).

The computational domain used is a square of side $\mathrm{L}=7 \delta_{i}$, corresponding to the most amplified wave number $\alpha_{a}=0.889 \delta_{i}^{-1}$ given by the linear stability theory. The Reynolds number is 300 , the Prandtl number is 1 and the Richardson number tested are $0.0,0.1$ and 0.2 , respectively. The initial amplitude of the perturbation was $10^{-6} U$. As the size domain is $7 \delta_{i}$ only the development of a single $\mathrm{KH}$ billow happened, excluding the possibility of paring process.

Tests with different computational grids of $n_{x} \times n_{z}$ points were done (see Tab.1). In these tests the vertical diffusion term, corresponding to the streamwise velocity was canceled. This diffusion increases the width of the shear layer during the simulation and implies a variation in time of the base flow, inducing variation of the amplification rate (Medeiros et al., 2002).

As expected, the grid size has a great influence over the amplification rate. In the test with a computational grid of $N 2=64 \times$ 129 points, it was noticed that the streamwise resolution interferes in the evolution of the wave amplitude (stratified case), when comparing with grid $N 1$ (see Tab.1). Thus, for the stratified case ( $R i$ $=0.1)$ the grid $N 2$ showed a decrease of the amplification rate due to the increase in the vertical resolution, with a error of $7 \%$ in relation to the reference value, whereas for $R i=0.2$ there is an increase in the amplification rate. Probably this occurs because the streamwise density gradient is not being well solved.

Figure 2 shows the time evolution of the amplitude for different Richardson numbers $(0 ; 0.1 ; 0.2)$ obtained from the simulation with the grid $N 3=128 \times 129$ points. Clearly, there is a region of exponential amplification, which corresponds to the regime governed by the linear theory.

In this test, the errors found are of $-0.32 \%$ for $R i=0,3.5 \%$ for $R i=0.1$ and $10.2 \%$ for $R i=0.2$. The comparison of the simulation
N3 with the numerical results of Hazel (1972), shown in Tab.1, gives good agreement for the temporal growth rates the stably stratified mixing layer.

Table 1. Comparison of amplification rate with the reference value or different grids.

\begin{tabular}{|l|c|c|c|c|}
\hline \multicolumn{5}{|c|}{ Amplification rate } \\
\hline $\mathrm{Ri}$ & $\begin{array}{c}\mathrm{N} 1 \\
64 \times 65\end{array}$ & $\begin{array}{c}\mathrm{N} 2 \\
64 \times 129\end{array}$ & $\begin{array}{c}\mathrm{N} 3 \\
128 \times 129\end{array}$ & $\begin{array}{l}\text { Ref. Value } \\
\text { Hazel (1972) }\end{array}$ \\
\hline 0 & 0.18732 & 0.18632 & 0.1861 & 0.1867 \\
\hline 0.1 & 0.19492 & 0.17057 & 0.1650 & 0.1594 \\
\hline 0.2 & 0.13597 & 0.16532 & 0.1329 & 0.1259 \\
\hline
\end{tabular}

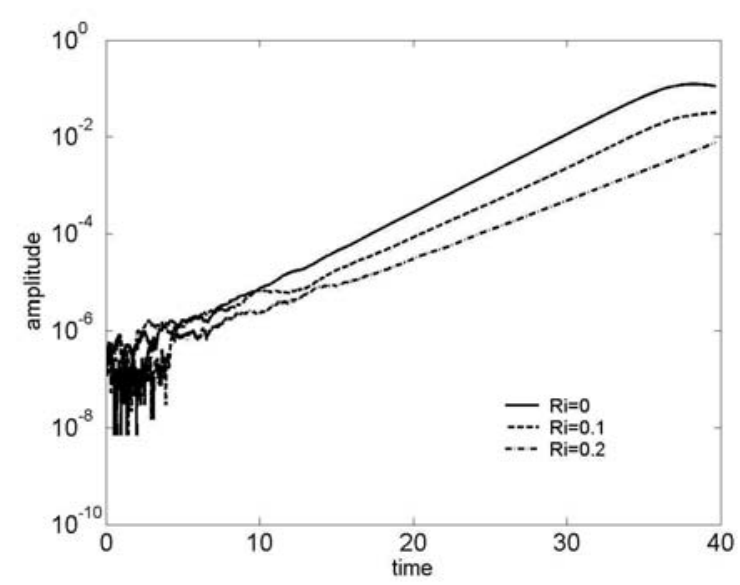

Figura 2. Amplitude evolution for simulation N3.

Two-Dimensional Visualizations - the Formation of the Baroclinic Layer

In the following section, results from 2D simulations are presented. Here the computational domain is $\left(L_{x}, L z\right)=\left(28 \delta_{i}, 28 \delta_{i}\right)$, with a computational grid of $256 \times 257$ points, $R e=300$ and $R i=0 ; 0.1 ; 0.2$. The amplitude of the perturbation superimposed upon the basic velocity profile, for the fundamental and the subharmonic mode, is $1 \% U$ and $0.1 \% U$, respectively.

Firstly, the evolution of a non-stratified mixing layer is analyzed. The initial vorticity, which is modulated by a small perturbation, progressively accumulates in the cores of the $\mathrm{KH}$ billows (Fig. 3). These cores are unstable to perturbation of wavenumber equal to $\alpha_{a} / 2$. The growth of this subharmonic perturbation leads to the pairing of the two vortices (Fig. $3 \mathrm{c}$ and Fig. $3 d)$.

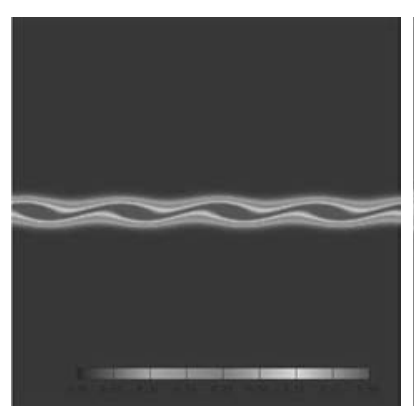

(a)

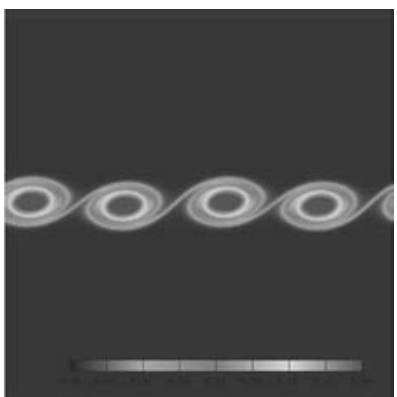

(b)
Figure 3. Spanwise vorticity fields, for a non-stratified case, $\mathbf{R i}=0$, at times: (a) 9.52; (b) 19.03; (c) 28.55 and (d) 38.07 


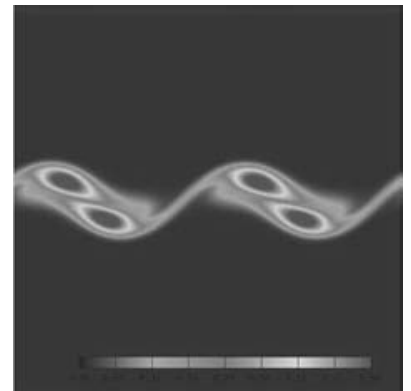

(c)

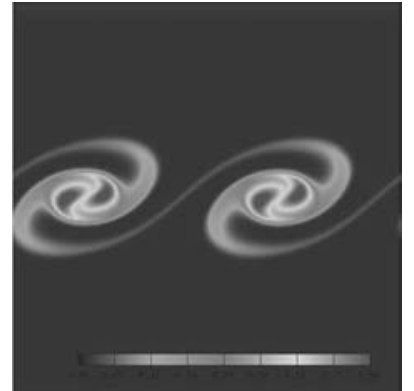

(d)
Figure 3. (Continued).

In a mixing layer, the primary $\mathrm{KH}$ vortices are not totally isolated from each other, but they are rather connected by a thin braid of spanwise vorticity. In a stably stratified mixing layer, these thin vorticity layers are strained in between the $\mathrm{KH}$ vortices and intensified by the buoyancy effects (Fig. 4b and Fig. 5b). As the development of the $\mathrm{KH}$ instability on a stably stratified mixing layer proceeds, a streamwise density gradient occurs in between the $\mathrm{KH}$ vortices, at the location where the braids form. This streamwise density gradient (corresponding to the spanwise component of the baroclinic torque, in the Boussinesq approximation) feeds the braid with vorticity and forms the baroclinic layer. This layer is associated with a strong density gradient and vorticity field. The baroclinic layer, as it is referred to in Staquet (1995), does not exist in an nonstratified flow. It forms under the action of buoyancy effects and strains between the KH vortices. The component of the baroclinic torque along the spanwise direction is showed below in the vorticity equation,

$$
\frac{\partial \omega_{y}}{\partial t}+u \frac{\partial \omega_{y}}{\partial x}+w \frac{\partial \omega_{y}}{\partial z}=R i \frac{\partial \rho}{\partial x}+\frac{1}{R e}\left(\frac{\partial^{2} \omega_{y}}{\partial x^{2}}+\frac{\partial^{2} \omega_{y}}{\partial z^{2}}\right) .
$$

The density gradient source term contribute as an extra mechanism for the generation or destruction of local vorticity by means of the baroclinic torque. This property yields a different behavior of the stratified mixing layer as opposed to its nonstratified counterpart. It follows that vorticity can locally increase beyond its maximum value for the stratified case.

Two secondary instabilities are propagated in the stratified mixing layer caused by the streamwise density gradient: the gravitational convective instability and the secondary shear instability. The first is found within unstable regions of the KH core, which consist of heavy and light fluid wrapped in a spiral roll. Wherever heavy fluid is found on top of light fluid, the gravitational convective instability amplifies due to buoyancy forces.

During the roll-up of $\mathrm{KH}$ billows, heavy and light fluid are brought together from the outer-side of the mixing layer towards the center of mixing layer, yielding a strong density gradient there. In this way, the secondary shear instability induced by the streamwise density gradient concentrates the vorticity in the baroclinic layer. The baroclinic layer is observed to occur in our simulations (Fig. 4d and Fig. 5d).

Figure 4 and 5 show the development of the Kelvin-Helmholtz instability, in the stratified mixing layer, for the same characteristic times.

These pictures show that when the Richardson number is increased the pairing process is inhibited and the braid vorticity exceeds the core's one. The stable stratification, through streamwise variations of the density field, weakens the vertical motions and reduces the buoyancy flux, as it can be observed in Fig. 6 .
In the non-stratified case, $R i=0$, it is observed that the vorticity remaining in the core is substantially greater than that in the braids. On the contrary, when the stratification is higher $(R i=0.2)$ the vorticity is increased in the braids and the baroclinic layer is visible, as can be seen in Fig. 5 d.

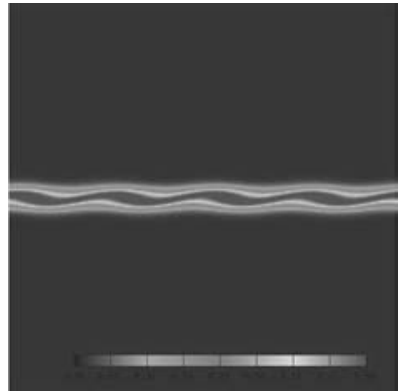

(a)

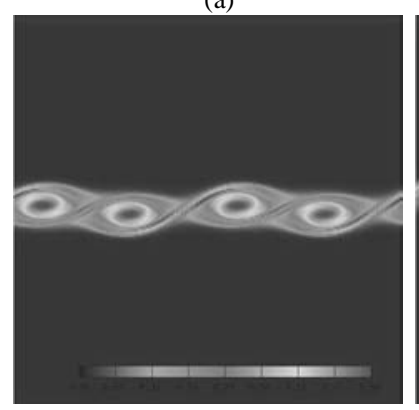

(c)

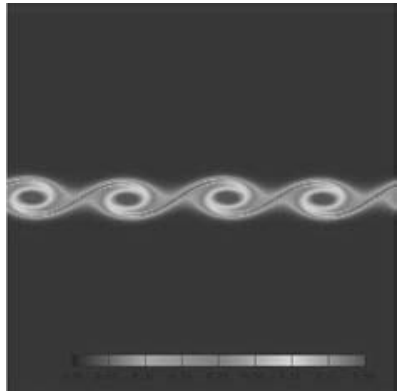

(b)

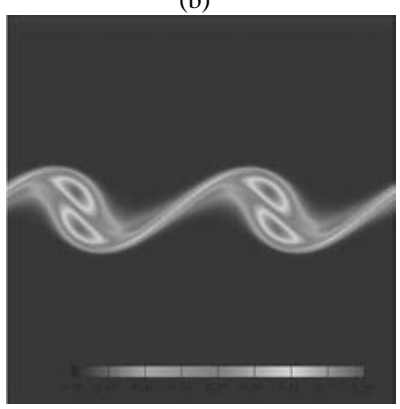

(d)
Figure 4. Spanwise vorticity fields, stratified case, $\mathbf{R} \mathbf{i}=\mathbf{0 . 1}$, in times: (a) 9.52; (b) 19.03 ; (c) 28.55 and (d) 38.07 .

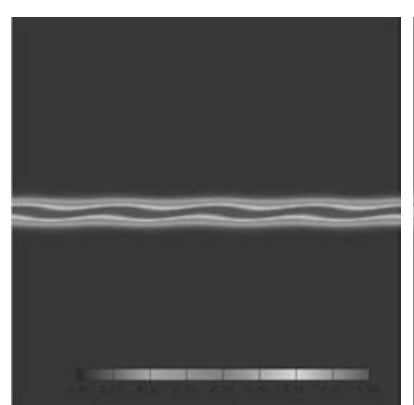

(a)

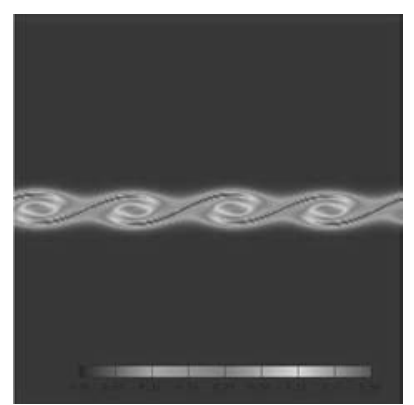

(c)

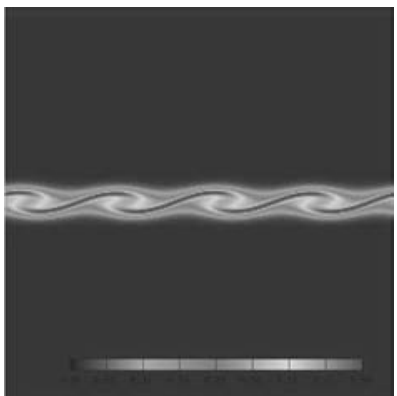

(b)

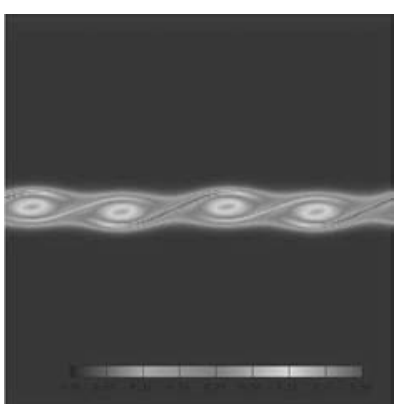

(d)
Figure 5. Spanwise vorticity fields, stratified case, $\mathbf{R} \mathbf{i}=\mathbf{0 . 2}$, in times: (a) 9.52; (b) 19.03 ; (c) 28.55 and (d) 38.07 . 


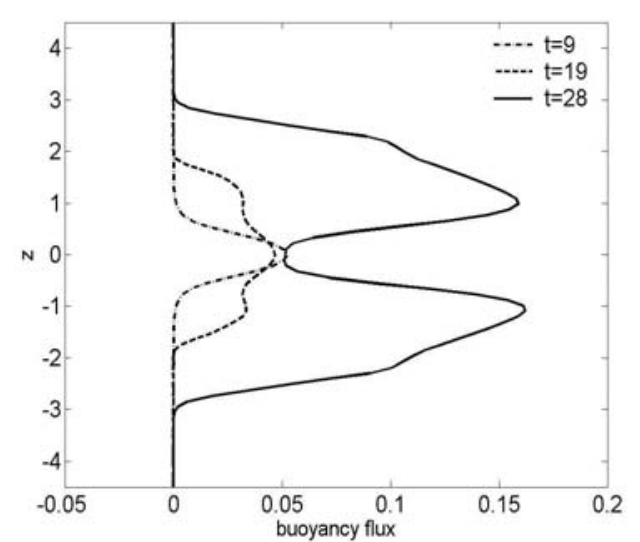

(a)

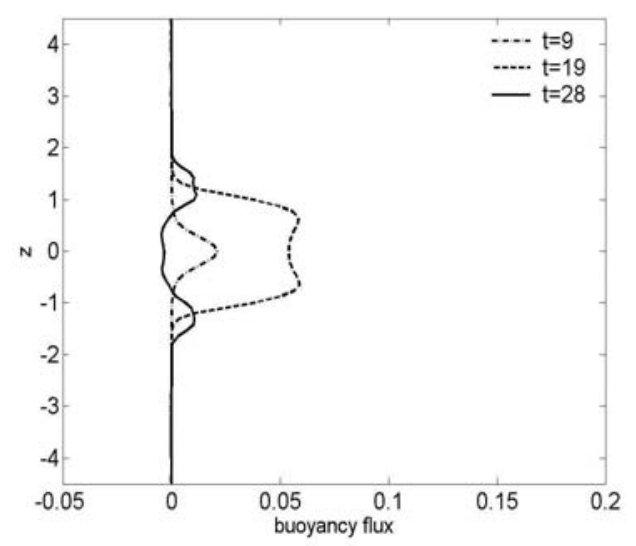

(b)

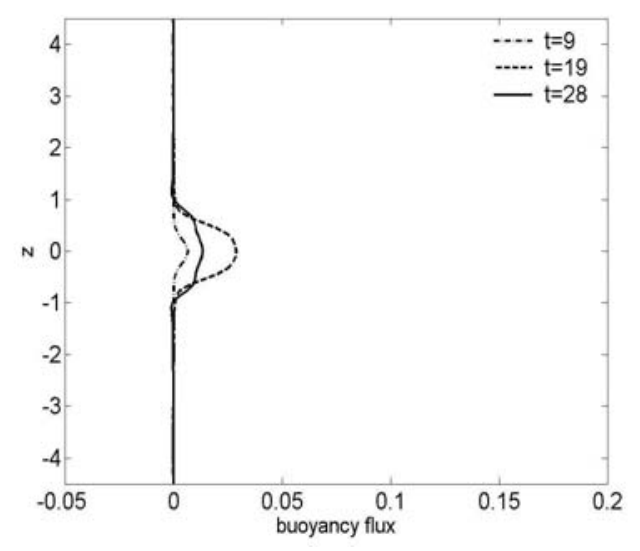

(c)

Figure 6. Buoyancy flux, $\left\langle\rho^{\prime} w\right\rangle$, at different times, $\mathbf{R e}=300$. (a) $\mathbf{R i}=0$; (b) $\mathbf{R i}=0.1$; (c) $\mathbf{R i}=0.2$.

The spreading of the buoyancy flux $\left\langle\rho^{\prime} w^{\prime}\right\rangle$ (Fig. 6) is due to the buoyancy effects. In the non-stratified case, there is no buoyancy force and the density is a perfect passive scalar (Fig. 6a). At time $\mathrm{t}=$ 28.55 the vortices are dislocated in relation to the $z$-center of the domain (Fig. 6b), producing negative values of the $\left\langle\rho^{\prime} w^{\prime}\right\rangle$. Figure $6 \mathrm{~b}$ and Fig. 6c show that there is a strong decrease of the buoyancy flux caused by increasing the Richardson number. This drastic reduction of the buoyancy flux as the Richardson number increases causes the reduction of fluid entrainment into $\mathrm{KH}$ vortices and delays pairing process of the large structures.

Therefore, the stable stratification has a stabilizing effect on the growth of the primary KH instability (Fig. 4 and Fig. 5), resulting in a decrease of the overall fluid entrainment, a reduction the mixing process and a decrease of the thickness of the mixing layer. Figure 7 shows the time evolution of the thickness of the mixing layer for different Richardson numbers.

To quantify the effect that a stable stratification (buoyancy forces) has on the development of KH instability, the time evolution of the kinetic energy was calculeted. The kinetic energy per unit area is given by

$$
K=\frac{1}{L_{x} L_{z}} \int_{0}^{L_{z}} \int_{0}^{L_{x}}\left((u-\langle u\rangle)^{2}+\left(w^{\prime}\right)^{2}\right) d x d z,
$$

where the ensemble average of the streamwise velocity is

$$
\langle u\rangle=\frac{1}{L_{x}} \int_{0}^{L_{x}} u d x .
$$

Figure 8 shows the evolution of the kinetic energy for different Richardson numbers. The peak of the maximum kinetic energy is attained for the first pairing (non-stratified and stratified cases), and then it oscillates and increases near the second pairing for the nonstratified case. It seems clear from Fig. 8 that the maximum $\mathrm{KH}$ vorticity amplitude is limited by the presence of a strong stable stratification $(R i \geq 0.1)$ in the flow and that the time at which the maximum energy is reached is considerably delayed when the stratification is increased. These characteristics are consistent with the expectation that the wave needs to execute work against the gravitational potential in order to grow.

\section{Three-Dimensional Visualizations - The Formation of}

\section{Streamwise Vortices}

The computational domain is a parallelepipedic box, of side 14 $\delta_{i}$ along the longitudinal $(x)$ and vertical $(z)$ directions and of side $10.5 \delta_{i}$ along the spanwise $(y)$ direction. All the three-dimensional tests were carried out with the resolution $(128,96,129)$ along the $(x$, $y, z$ ) directions, respectively.

The spanwise length of the domain, $L_{x}$, is $2 / 3$ of $L_{y}$. This choice was made to force the most amplified mode in the spanwise direction predicted by Pierrehumbert and Widnall (1982). The parameters used in the tests are the Reynolds number, $R e=200$, the Richardson number, $R i=0$ (non-stratified case), 0.1 and 0.2 (strong stratification) and Prandtl number, $\operatorname{Pr}=1$.

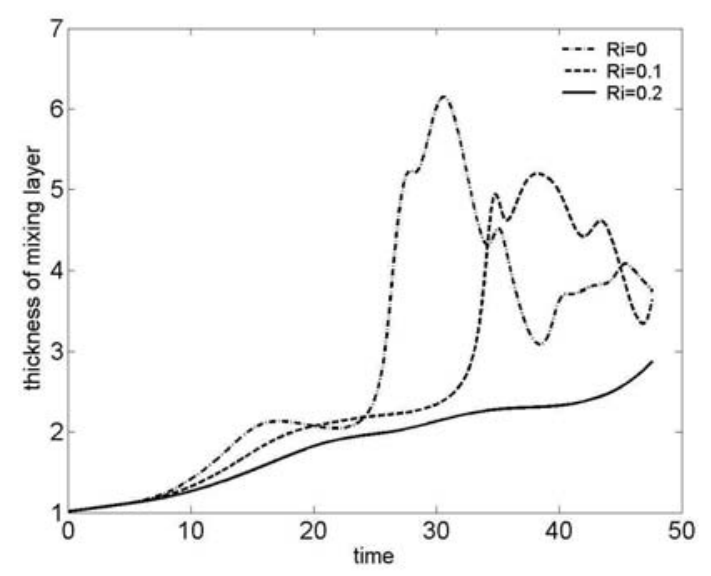

Figure 7. The effect of the bulk Richardson number on the evolution of the thickness of the mixing layer, $\mathrm{Re}=\mathbf{3 0 0}$.

\section{J. of the Braz. Soc. of Mech. Sci. \& Eng.}




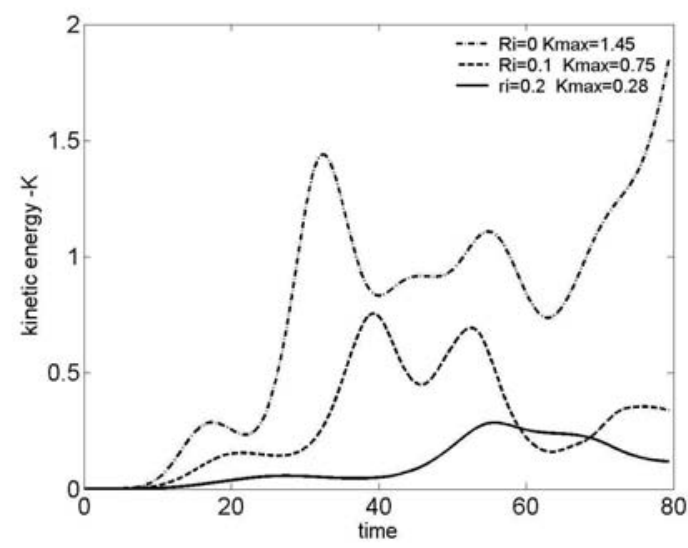

Figure 8. The effect of the bulk Richardson number on the evolution of the wave kinetic energy, $R e=300$.

Three-dimensional visualizations are presented for 3 different Richardson numbers for the same instant of time, with isosurfaces of vorticity modulus $|\omega|=0.25 \omega_{i}$, where $\omega_{i}=2 U / \delta_{i}$. The amplitude of the perturbation (forced) superimposed upon the basic velocity profile, for the fundamental, the subharmonic and spanwise mode is $1 \% U, 0.1 \% U$ and $0.1 \% U$, respectively.

The initial condition is the same for all the numerical tests. At time $\mathrm{t}=9.52$ only the non-stratified mixing layer $(R i=0)$ was unstable, showing the formation of two longitudinal wavelength, characterizing the Kelvin-Helmholtz instability (Fig. 9).

At time $\mathrm{t}=19.02$, for the non-stratified case, $R i=0$, there are two primary billows $\mathrm{KH}$ (spanwise vortices), due to the $\mathrm{KH}$ instability and fundamental mode, whereas for the stratified case o roll-up occurs $(R i=0.1)$ and 2D instability is formed in the vorticity layer $(R i=0.2)$. This occurs because the stratification affects the vertical movements, diminishing the intensity of the oscillations and reducing the kinetic energy, thus delaying the formation of the $\mathrm{KH}$ structures, as it can be verified in the $2 \mathrm{D}$ simulation above and in Martinez et al. (2004).

Figure 10 shows the results obtained for $R i=0 ; 0.1 ; 0.2$ at times 57.14 and 76.19. In the non-stratified case, at time $t=76.19$, streamwise vortices are clearly formed. The two pairs of counterrotating streamwise vortices appear between the times 47.62 and 57.14 , one pair for each fundamental wavelength. The streamwise vortices, or ribs, show a high degree of coherence and are extend with nearly the same vortical intensity over the complete braid region.

In the stratified cases, on the other hand, the secondary instability dominates the three-dimensionalization of the layer and the streamwise vortices are now seen to be less developed over the complete domain. This is due to streamwise density gradient that decreases the levels of vorticity in the $\mathrm{KH}$ billow while increases it in the braid zone. This effect is easier to see in highly stratified flows, $R i=0.2$, than in "mildly" stratified flow, $R i=0.1$.

Figure 10 shows the translative instability acting on the resultant vortex of the pairing process, whereas for $R=0.2$ the flow is still two-dimensional. The three-dimensionalization of the mixing layer is dominated by the initial forcing condition of the sub harmonic and spanwise modes. As sub harmonic mode grows before the spanwise mode, the formation of the streamwise vortices is delayed. The stratification also delays the pairing process and development of the translative instability. This fact can be observed when comparing the non-stratified case with the stratified one in Fig. 9 and 10 .
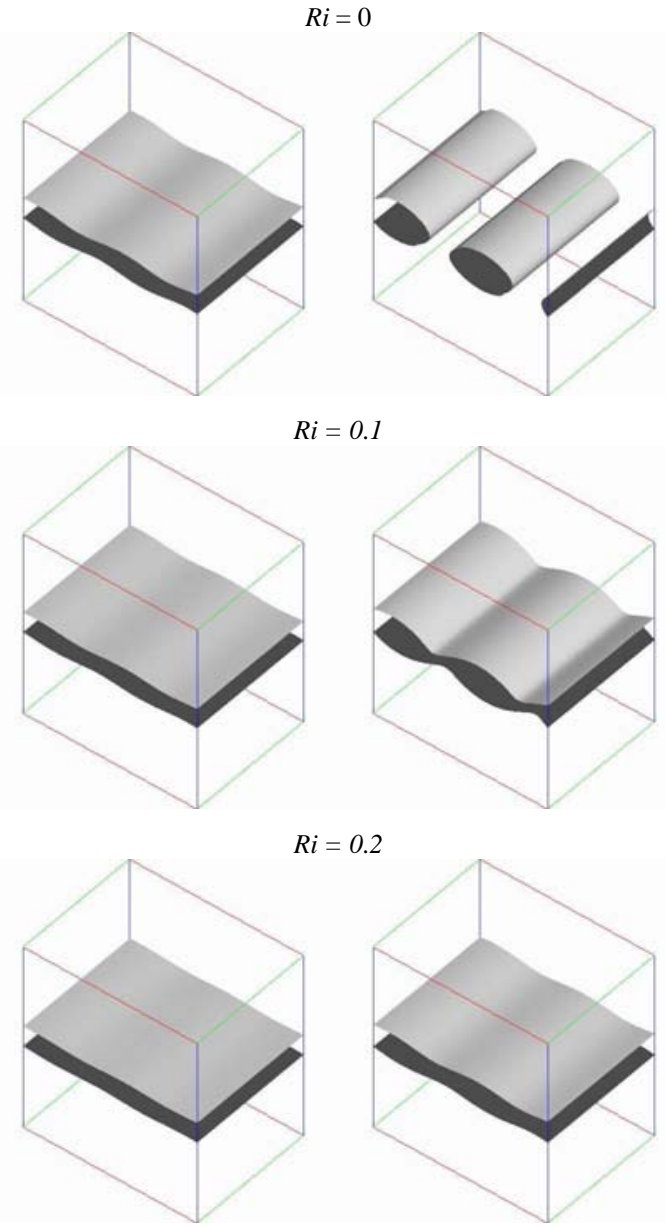

Figure 9. Isosurfaces of vorticity modulus at $t=9.52$ and $19.02, R e=200$, $|\omega|=0.5$.

Figure 11 shows the isosurface of vorticity modulus at time 104.77 and 114.29 , for the two stratified cases considered. In the test with $R i=0.1$ a deformation in the structure of the streamwise vortices is observed. This deformation, probably caused by baroclinic effects, can be originated by the translative instability occurring in the region between the billows and not in its core, as in the non-stratified case (Caulfield and Peltier, 2000).

For $R i=0.2$ the stabilizing effect of the stratification becomes strongly visible in the reduction of the amplitude of the $3 \mathrm{D}$ disturbances. It is an indication of the qualitative variation of the dynamics of the flow, where buoyancy forces inhibit the growth of the turbulent kinetic energy, mainly the vertical speed. This effect can be observed in Fig. 12, which indicates that when the Richardson number increases the buoyancy flux decreases.

To be able to have the best possible picture of the vortices spatial structure the Q criterion was used (Dubief and Delcayre, 2000; Jeong and Hussain, 1995). It is well known that regions of high vorticity often correspond to coherent structure locations, or even sheared zones without any structures. The $\mathrm{Q}$ criterion is defined by:

$Q=-\frac{1}{2} \frac{\partial u_{i}}{\partial x_{j}} \frac{\partial u_{j}}{\partial u_{i}}=\frac{1}{2}\left(\|\Omega\|^{2}-\|S\|^{2}\right)=\frac{1}{2} \nabla^{2} P$

where $\mathrm{i}, \mathrm{j}=1,2,3$ and $\|\Omega\|^{2}$ is the rotation rate and $\|S\|^{2}$ the strain rate. 

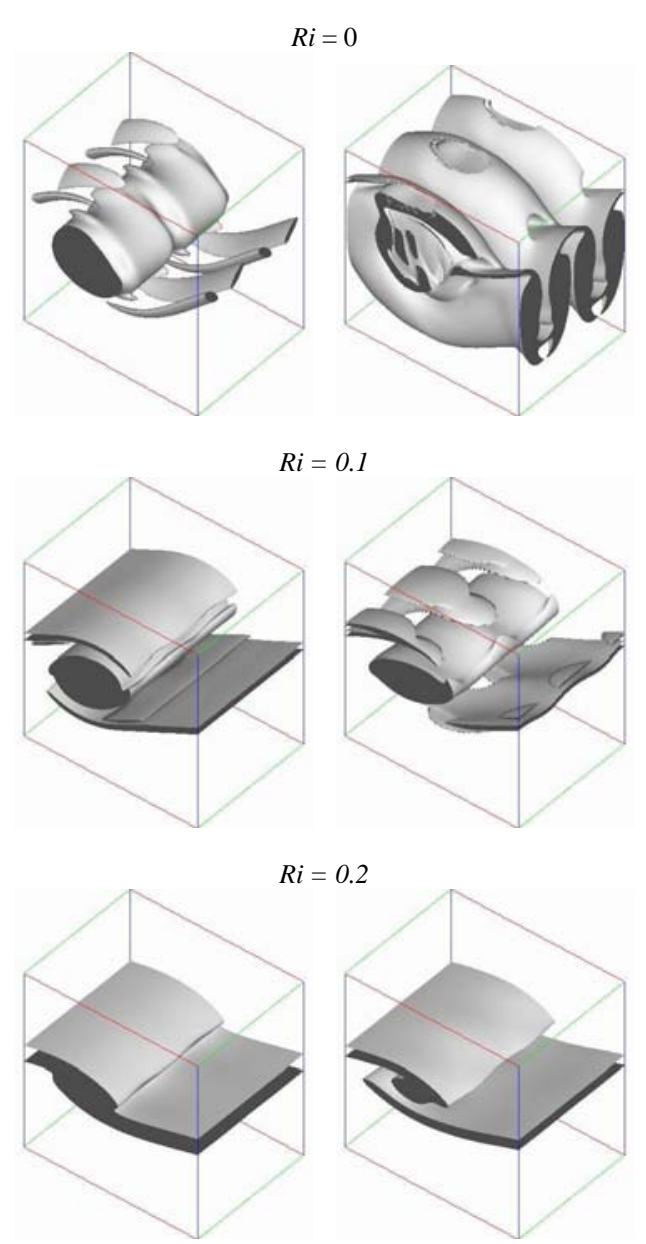

Figure 10. Isosurfaces of vorticity modulus at $\mathrm{t}=\mathbf{5 7 . 1 4}$ and $\mathbf{7 6 . 1 6}, \mathbf{R e}=$ 200, $|\omega|=0.5$.
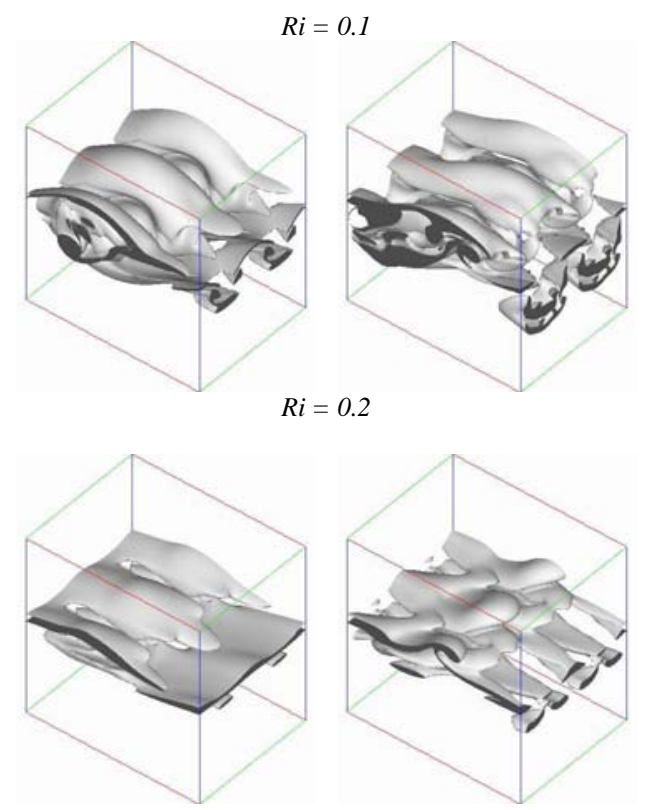

Figure 11. Isosurfaces of vorticity modulus at $\mathrm{t}=104.77$ and $114.29, \mathbf{R e}=$ 200, $|\omega|=0.7$.

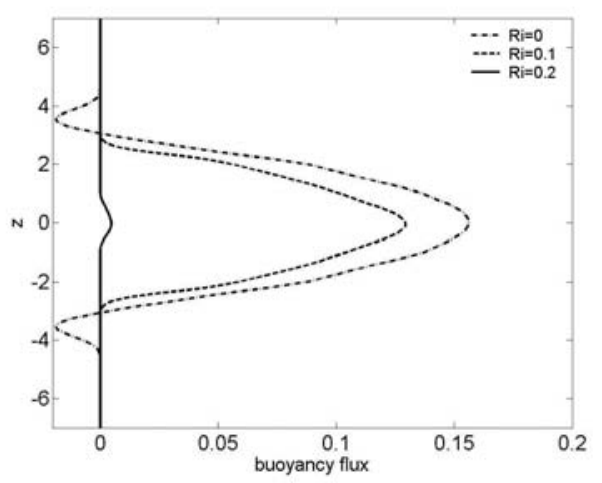

(a)

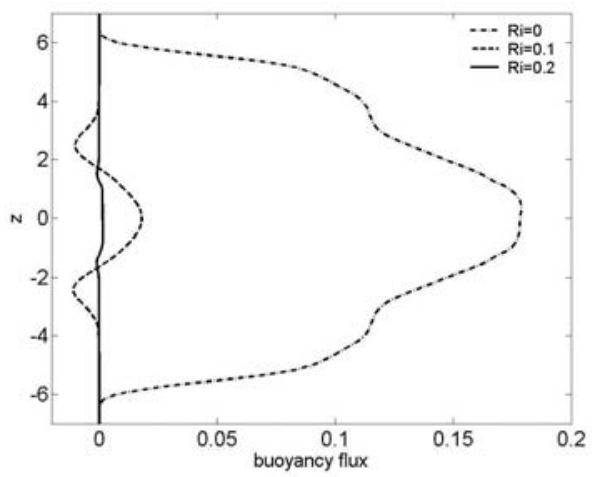

(b)

Figure 12. Buoyancy flux $\left\langle\rho^{\prime} w^{\prime}\right\rangle$, at $R i=0 ; 0.1 ; 0.2, R e=200$, at time. (a) 38.09; (b) 76.19 .

Vorticity modulus visualization emphasizes intermediate scale vortices, though it may obscure the large structures. The positive $\mathrm{Q}$ values occur in flow regions where local rotation is predominant, especially in regions associated with the vortex cores.

In the $\mathrm{Q}$ criterion, a balance between the rotation rate and strain rate was done. This implies that positive $\mathrm{Q}$ isosurfaces isolate areas where the strength of rotation overcomes the strain. In Fig. 13, it can be observed a comparison between isosurfaces of vorticity modulus and Q-isosurfaces for the non-stratified case, at time $\mathrm{t}=76.19$, and for the stratified cases at times $\mathrm{t}=104.77(R i=0.1)$ and $\mathrm{t}=114.29$ $(R i=0.2)$.

Figure 14 shows spanwise cross-sectional plots of the vorticity modulus for a domain of two fundamental streamwise wavelengths $\left(\lambda_{\mathrm{I}}\right)$, for one characteristic time and with forced initial conditions. The longitudinal variation of the domain varies is $0<x<14 \delta_{i}$. At $t$ $=76.19$, streamwise vortices are clearly formed in a non-stratified mixing layer while for the stratified cases only some concentration of vorticity may be identified, mainly for $R i=0.1$ at the center of the mixing layer. 


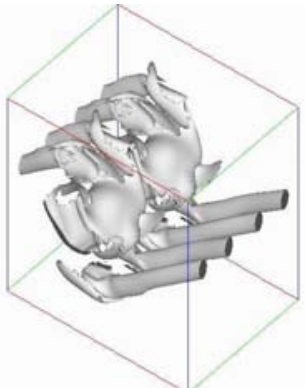

(a)

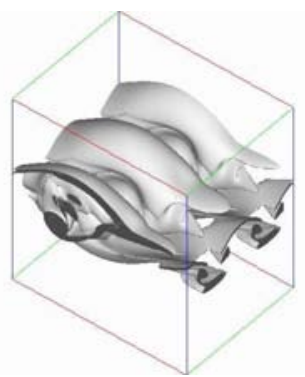

(c)

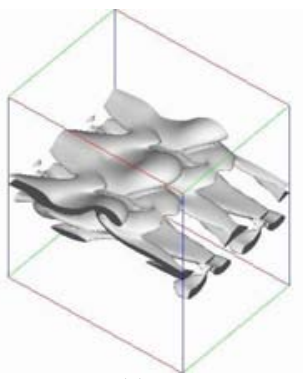

(e)

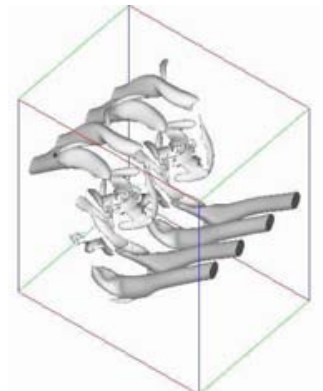

(b)

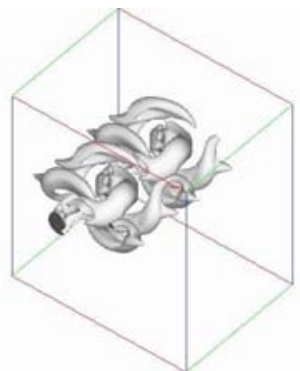

(d)

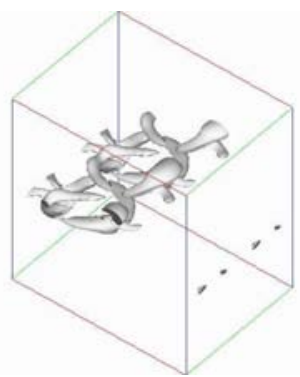

(f)
Figure 13. (a) Isosurfaces of $|\omega|=2.5, R i=0$; (b) $Q$ - isosurfaces $Q=1, R i$ $=0$; (c) Isosurfaces of $|\omega|=0.7, R i=0.1 ;(d) Q-$ isosurfaces $Q=0.2, R i=$ 0.1 ; (e) Isosurfaces of $|\omega|=0.7, R i=0.2$; (f) $Q-$ isosurfaces $Q=0.08, R i=$ 0.2

At a later time, $t=104.77$, Fig. 15 shows intense streamwise vortices for the two stratified cases considered. While their vorticity is lower than the non-stratified case, the formation mechanism seems to be related to the secondary shear instability.

Comparing the spanwise cross-sectional plots in Fig.14a for an non-stratified flow with those from Fig.14b and Fig.14c, we can remark how the entrainment process significantly influences the stratification: the vertical extent of the active region, roughly corresponding to that occupied by entrained fluid, has narrowed. This is also apparent from the development of the size of the mixing layer for different $R i$, where entrainment is progressively suppressed with increasing stratification. Therefore, in stratified cases the longitudinal structures are confined in a shorter vertical length that for an non-stratified case, and they are not developed over the whole vertical domain.
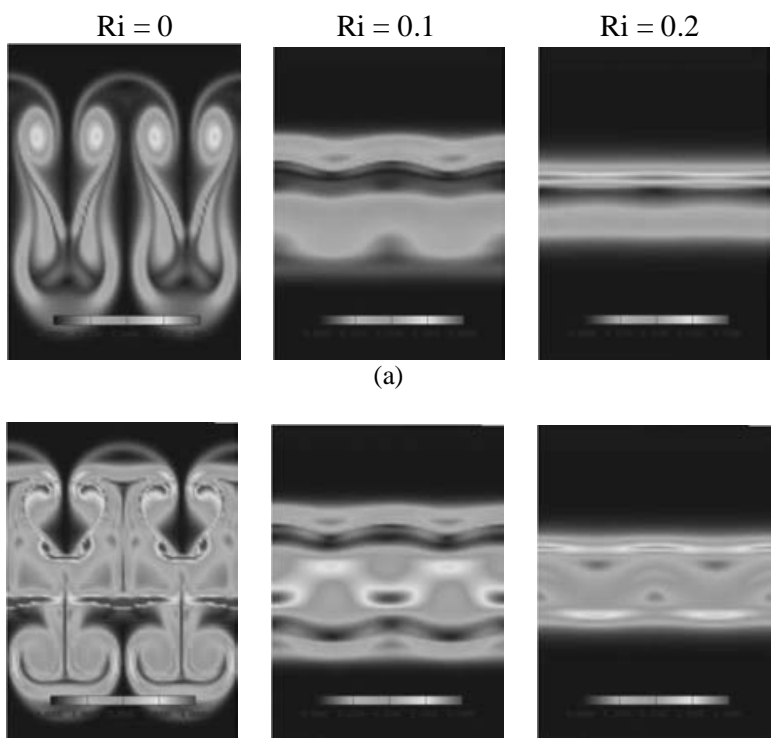

(b)
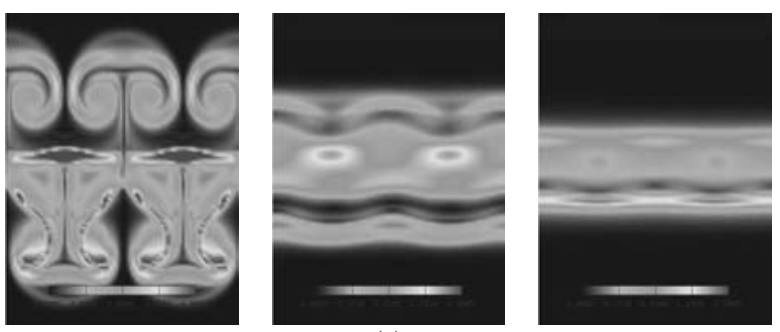

(c)
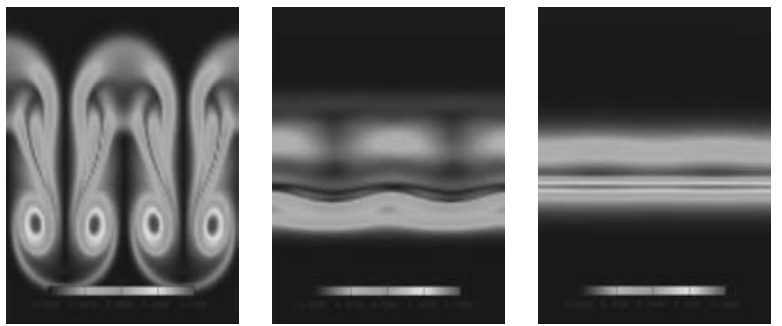

(d)
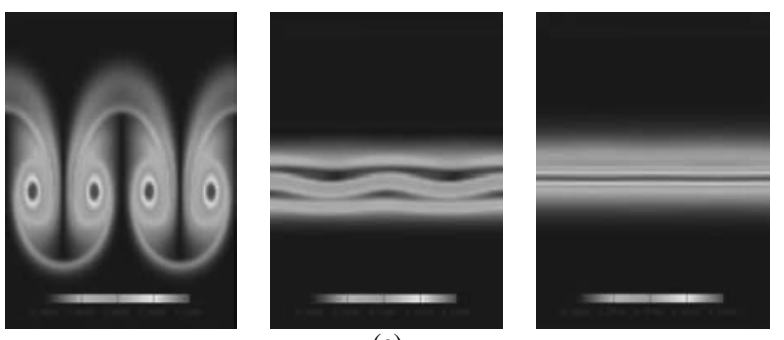

Figure 14. Spanwise cross-sectional plots of vorticity modulus at $x$ equal (a) 2; (b) 4; (c) 6; (d) 8 and (e) $10 \delta_{i}$, at time t $=76.19$. 

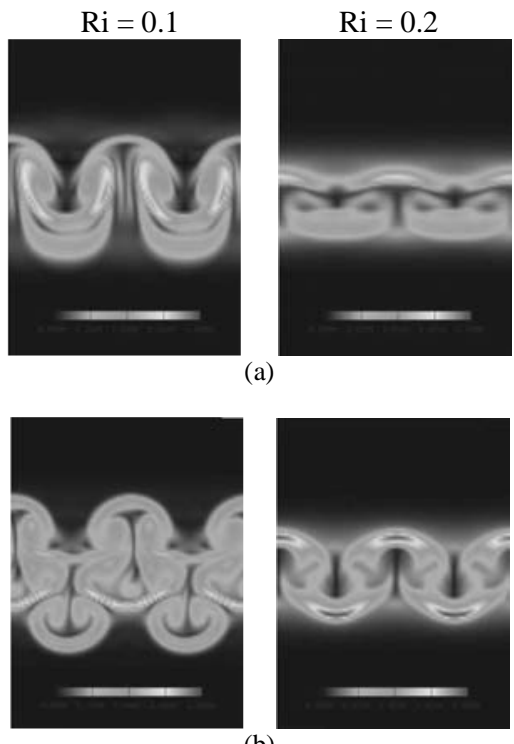

(b)
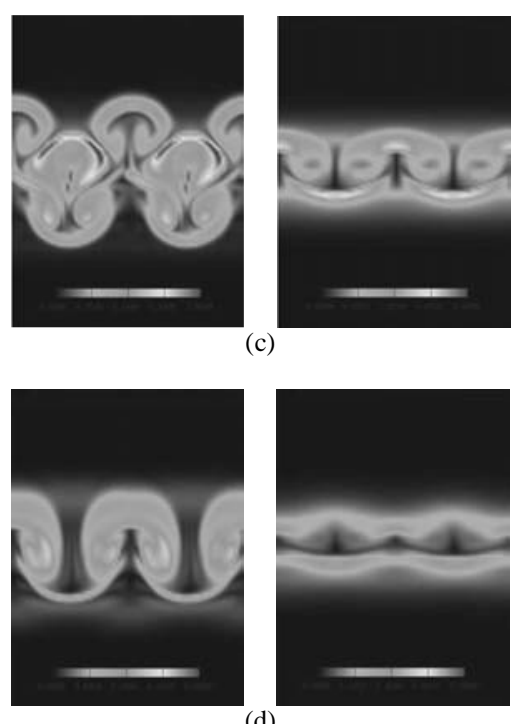

(d)
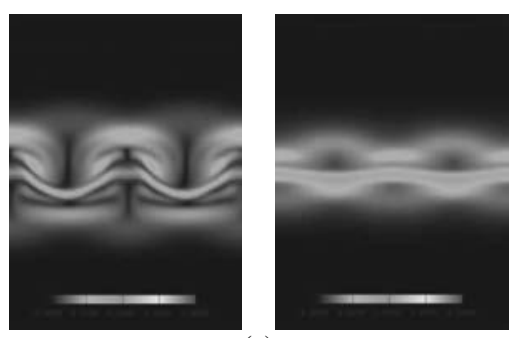

(e)

Figure 15. Spanwise cross-sectional plots of vorticity modulus at $x$ equal (a) 2; (b) 4; (c) 6; (d )8 and (e) $10 \delta_{i}$, at time $\mathrm{t}=104.77$.

\section{Conclusions}

The purpose of the present study was to investigate numerically the effect of the stable stratification (buoyancy forces) on the development of the $\mathrm{KH}$ instability and the formation of streamwise vortices, which are developed after the saturation of the primary billows of KH. The 2D simulations showed that higher stratification increasingly inhibits the pairing process, reduces the exchange of energy between the KH vortices and the flow, limits the maximum $\mathrm{KH}$ wave amplitude and reduces the buoyancy flux. The 3D simulations, with forced conditions, showed that streamwise vortices are clearly formed in a non-stratified mixing layer. The streamwise vortices, or ribs, showed a high degree of coherence and are spread with nearly the same vortical intensity over the complete braid region. On the other hand, in the stratified cases the secondary shear instability dominates the three-dimensionalization of the layer. This is due to the streamwise density gradient which decreases the levels of vorticity in the $\mathrm{KH}$ billow while increases in the braid zone. This effect is easier to be seen in highly stratified flows, $R i=$ 0.2 , than in a "middly" stratified flow, $R i=0.1$.

\section{Acknowledgments}

The first author acknowledges the financial support received from CAPES-PICDT.

\section{References}

Caulfield, C. P., and Peltier, W. R. 1994. "Three-dimensionalization of the stratified mixing layer”. Phys. Fluids, Vol.6, pp.3803-3805.

Caulfield, C. P., and Peltier, W. R. 2000. "The anatomy of the mixing transition in homogenuos and stratified free shear layers". J. Fluid Mech., Vol.413, pp.1-47.

Comte, P., Lesieur, M., and Lamballais, E. 1992. "Large and small-scale stirring of vorticity and a passive scalar in a 3-d temporal mixing layer". Phys. Fluids A, Vol.4 ,pp. 2761-2778.

Comte, P., Silvestrini, J. H., and Bérgou, P. 1998. "Streamwise vortices in Large Eddy Simulation of mixing layers". Eur. J. Mech. - B/Fluids, Vol.17, pp.615-637.

Corcos, G. M., and Sherman, F. S. 1976. "Vorticity concentration and the dynamics of unstable free shear layers". J. Fluid Mech., Vol.73, part2, pp.241-264.

Cortesi, A. B., Yadigaroglu, G., and Banerjee, S. 1998. "Numerical investigation of the formation of three-dimensional structures in stablystratified mixing layers". Phys. Fluids, Vol.10, pp.1449-1473.

Dubief, Y., and Delcayre, F. 2000. "On Coherent-vortex identification in turbulence". J. Turbulence, Vol.1, n.11, pp.1-22.

Hazel, P. 1972. "Numerical studies of the stability of inviscid stratified shear flows". J. Fluid Mech., Vol.51, pp.39-61.

Howard, L. N. 1961. "Note on a paper of John W. Miles". J. Fluid Mech., Vol.10, pp.509-512.

Jeong, J., and Hussain, F. 1995. "On the identification of a vortex". J. Fluid Mech., Vol.285, pp.69-94.

Klaassen, G. P., and Peltier, W. R. 1991. "The influence of stratification on secondary instability in free shear layers". J. Fluid Mech., Vol.227, pp.71106.

Lardeau, S., Lamballais, E., and Bonnet, J. P. 2002. "Direct Numerical Simulations of a jet controlled by fluid injection". J. Turbulence, Vol.3

Lasheras, J. C., and Choi, H. 1988. "Three-dimensional instability of a plane free shear layer: an experimental study of the formation and evolution of streamwise vortices". J. Fluid Mech., Vol.188, pp.53-86.

Lele, S. K. 1992. "Compact finite difference schemes with spectral-like resolution”. J. Comp. Phys., Vol.103, 16-42.

Martinez, D. M. V., Schettini, E. B. C., and Silvestrini, J. H. 2004 "Transition to turbulence in a stable stratified temporal mixing layer through direct numerical simulation". In: Proceedings of the 10th Congress of Thermal Sciences and Engineering, ABCM.

Medeiros, M. A. F., Silvestrini, J. H., and Mendonça, M. T. 2002. "Using linear and non linear stability theory for evaluating code accuracy". In: Proceedings of the III Escola de Primavera de Transição e Turbulência, ABCM.

Metcalfe, R. W., Orszag, S. A., Brachet, M. E., Menon, S., and Riley, J. J. 1987. "Secondary instability of a temporally growing mixing layer". J. Fluid Mech., Vol.184, pp.207-243.

Michalke, A. 1964. "On the inviscid instability of the hyberbolic tangent velocity profile”. J. Fluid Mech., Vol.19, pp.543-556.

Miles, J. W. 1961. "On the Stability of heterogeneous shear flows". J. Fluid Mech., Vol.10, pp.496-508.

Moser, R., and Rogers, M. M. 1993. "The three-dimensional evolution of a plane mixing layer: pairing and transition to turbulence". J. Fluid Mech., Vol.247, pp.275-320. 
Patnaik, P. C., Sherman, F. S., and Corcos, G. M. 1976 "A numerical simulation of Kelvin-Helmholtz waves of finite amplitude". J. Fluid Mech., Vol.73, 215-240.

Pierrehumbert, R. T., and Widnall, S. E. 1982. "The two and three dimensional Instabilities of a spatially periodic shear flows". J. Fluid Mech., Vol.114, 59-82.

Rogers, M. M., and Moser, R. 1992. "The three-dimensional evolution of a plane mixing layer: the Kelvin-Helmholtz rollup”. J. Fluid Mech., Vol.243, 183-226.
Showalter, D. G., Atta, C. W. Van, and Lasheras, J. C. 1994. "A study of streamwise vortex struture in a stratified shear layer". J. Fluid Mech., Vol.281, pp.247-291.

Silvestrini, J. H., and Lamballais, E. 2002. "Direct numerical simulations of wakes with virtual cylinders". Int. J. Comp. Fluid Dyn. Vol.16, n.4, pp.305-314.

Staquet, C. 1995. "Two-dimensional secondary instabilities in a strongly stratified shear layer". J. Fluid Mech., Vol.296, pp.73-126.

Williamson, J. H. 1980. "Low-storage Runge-Kutta schemes". J. Comp. Phys., Vol.35, pp.48-56. 